Carbon-Carbon Nozzle

Extension Devielopment.in.

\title{
Support of In-Space and
}

Upper Stage Liquid Rocket

Engines

AIAA Propulsion and Energy Forum

July 12, 2017

AIAA-2017-5064

Paul $R$ Gradl

Peter G, Valentine NASA Marshaill

-Paul.R.Gradl@nasa.gov.

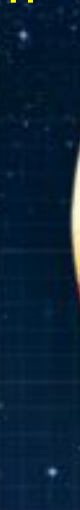




\section{Motivation for Extension Development}

- NASA and commercial space partners are interested in developing a commercial supply chain for Carbon-Carbon Nozzle Extensions (CCNE)

- Provides significant advantages for a variety of upper-stage engines and in-space engines

- Weight Reduction - $50 \%$ savings vs. metallic

- Improved thermal design margins $-500-1500^{\circ} \mathrm{F}$

- Less complex designs and/or manufacturing processes

- Cost Reduction

- New design opportunities to further optimize regen-extension joint

- Evaluate high temperature nozzle extension fabrication processes and obtain preliminary hot-fire test data in a relevant environment to characterize materials

Goal: Advance the state of the U.S. Carbon-Carbon (C-C) technology to the point that domestic $\mathrm{C}-\mathrm{C}$ nozzles can be considered as viable candidates for use on U.S. cryogenic upper stage engines, in-space, ascent/decent lander engines and nuclear engines 


\section{and Industry Partnerships}

\section{SBIR/STTR}

Development

A. PAN-based hybrid C-ZrC/C-C Ultramet, C-CAT

B. Rayon-based involute C-C MR\&D, Orbital ATK

C. PAN-based Ir-lined involute C-C MR\&D, Orbital ATK, Plasma Processes

D. PAN-based C-C with "high-melt" and SiC coating systems C-CAT

E. Lyocell-based C-C C-CAT, Southern Research

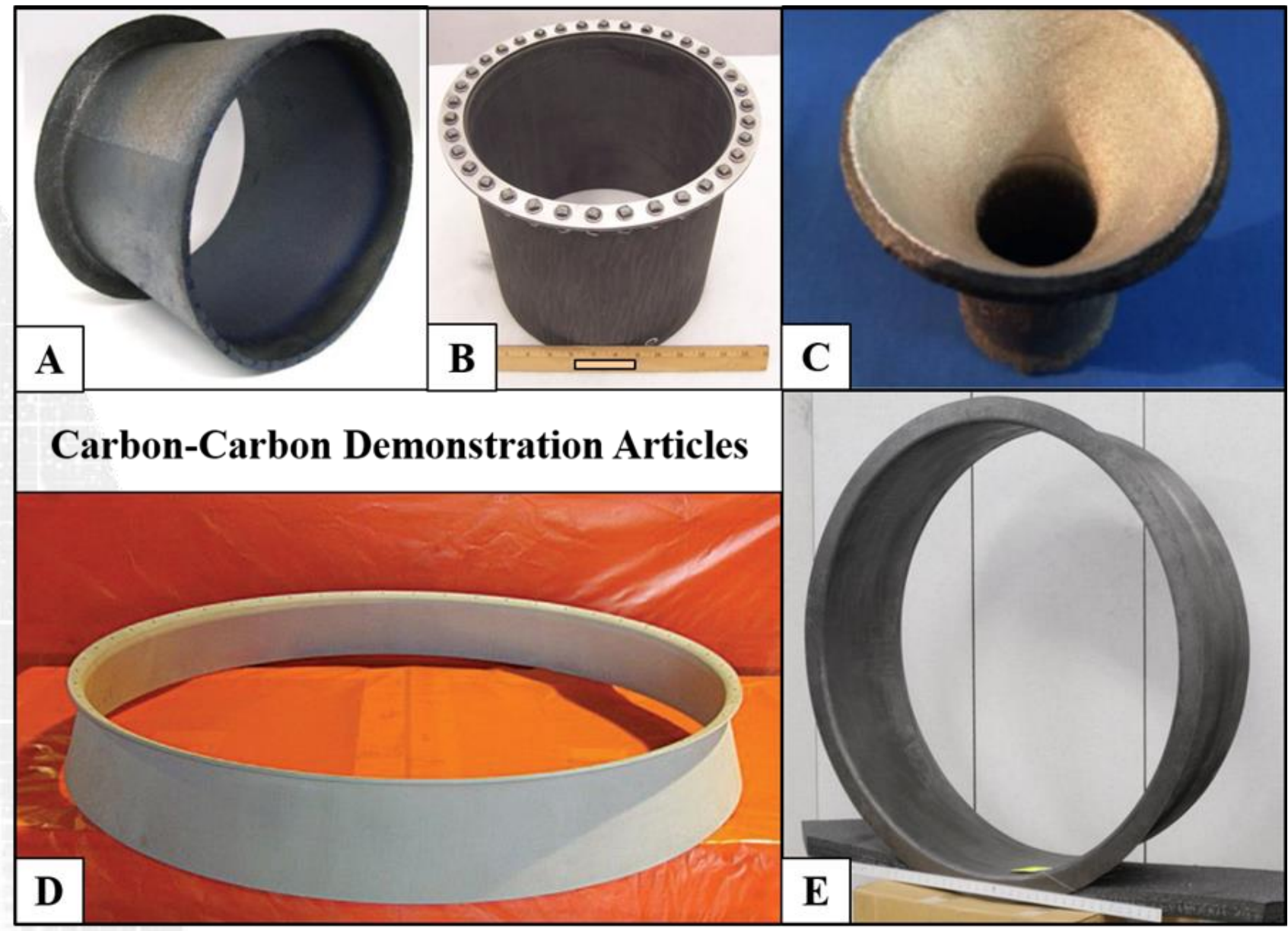

\section{MSFC In-House Technology Development Projects}

- Materials screening via $1.2 \mathrm{~K}-\mathrm{Ib}_{\mathrm{f}} \mathrm{LOX} / \mathrm{GH}_{2}$ small thruster testing

- Moderate-scale demonstration via 35K-lb $\mathrm{b}_{\mathrm{f}} \mathrm{LOX} / \mathrm{LH} 2$ chamber to evaluate material feasibility

- Component and coupon level material testing 


\section{Background of MSFC Test Rig}

- Starting in 2014, MSFC created a subscale nozzle test rig to conduct affordable, long-duration hot-fire testing for NASA and commercial partners

- LOX/GH2, LOX/CH4, or LOX/RP capabilities

- Durations up to 180 seconds

- Previous testing used a vintage chamber, which caused flow separation limiting the length of the nozzle

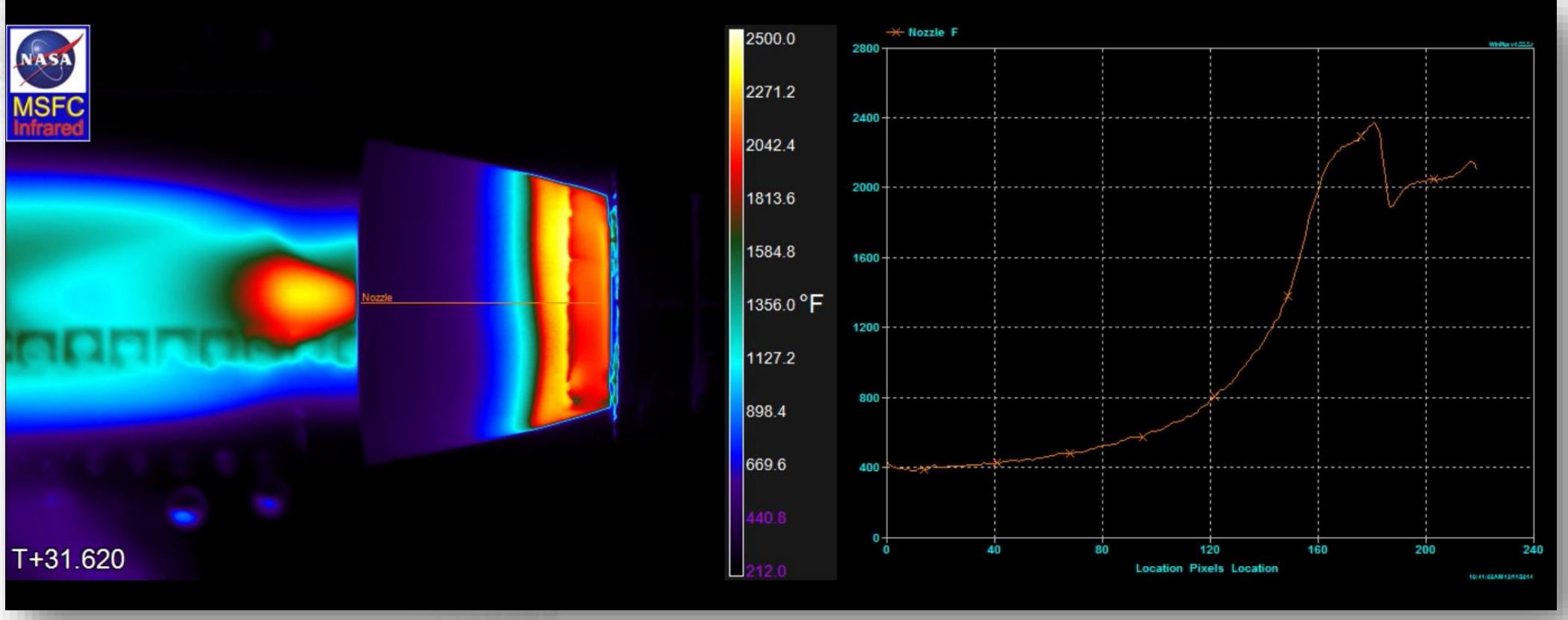




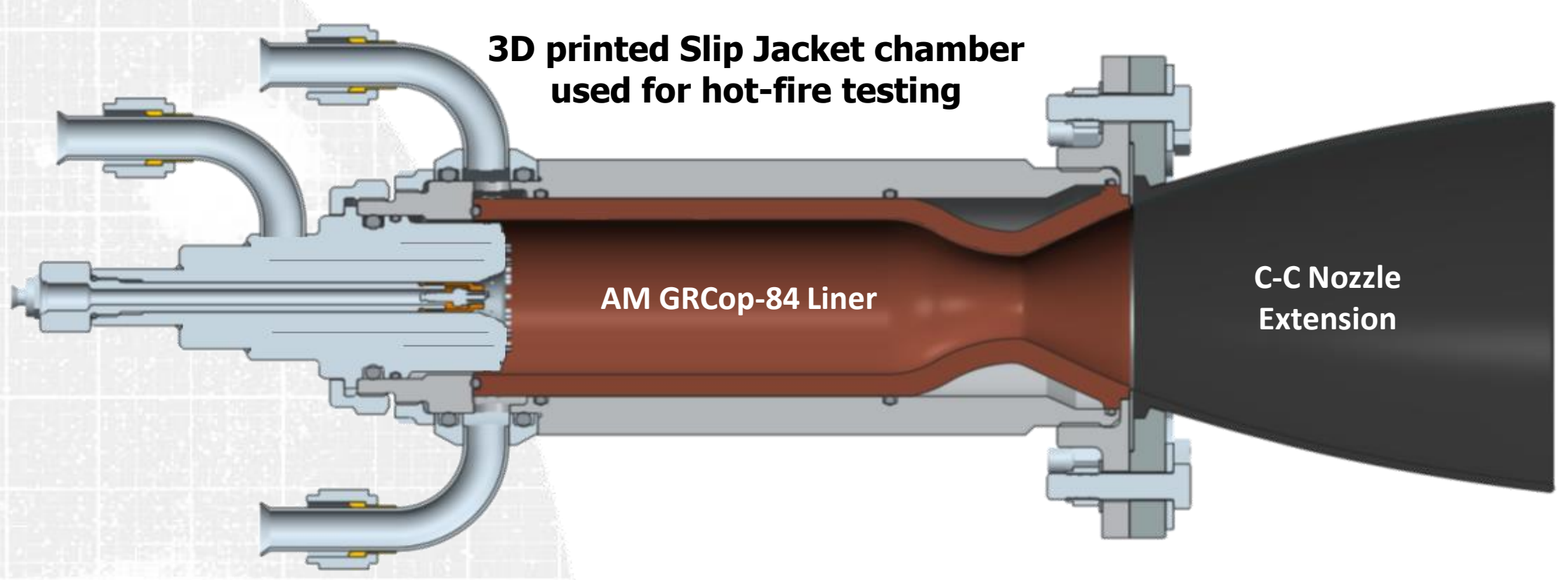

- New contour design allows for full-flow extended length nozzles and extensions

- 27:1 expansion

- Pc = 750 psig

- 1,200 lb $\mathrm{lf}_{\mathrm{f}}$ thrust

- Duration up to $180 \mathrm{sec}$

- Additive manufactured (AM) GRCop-84 liner

\begin{tabular}{lcc}
\hline \hline & Heritage Design & New Design \\
\cline { 2 - 3 } Thrust Chamber Assembly, Drawing Reference & MER00060-101 & MER01446-001 \\
Main Combustion Chamber, Liner & MED04227-1 & MER00664-001 \\
\hline Maximum Chamber Pressure, Pc (psia) & 850 & 1350 \\
Water Coolant Inlet Pressure, (psia) & 1000 & 2000 \\
Chamber Barrel Diameter (in) & 2.25 & 2.25 \\
Chamber Barrel Length (in) & 6.77 & 5.26 \\
Divergent Radius, Rd/Rt & 2 & 0.5 \\
Throat Diameter (in) & 1.2 & 1.2 \\
Nozzle Attach area ratio (AR) & $8.1: 1$ & $4.4: 1$ \\
\hline
\end{tabular}




\section{Orbital ATK CCNE Testing at MSFC TS115}

\section{Joint MSFC/OATK effort to demonstrate new test and material capabilities}

- Scale-up and demonstration of low cost manufacturing processes using tape wrapped preforms, a rapid densification process, and a variety of oxidation barriers.

- Static testing of extensions included:

- Demonstration of attachment and sealing concept for 2D CCNE's

- Demonstration of 2D C-C/oxidation-

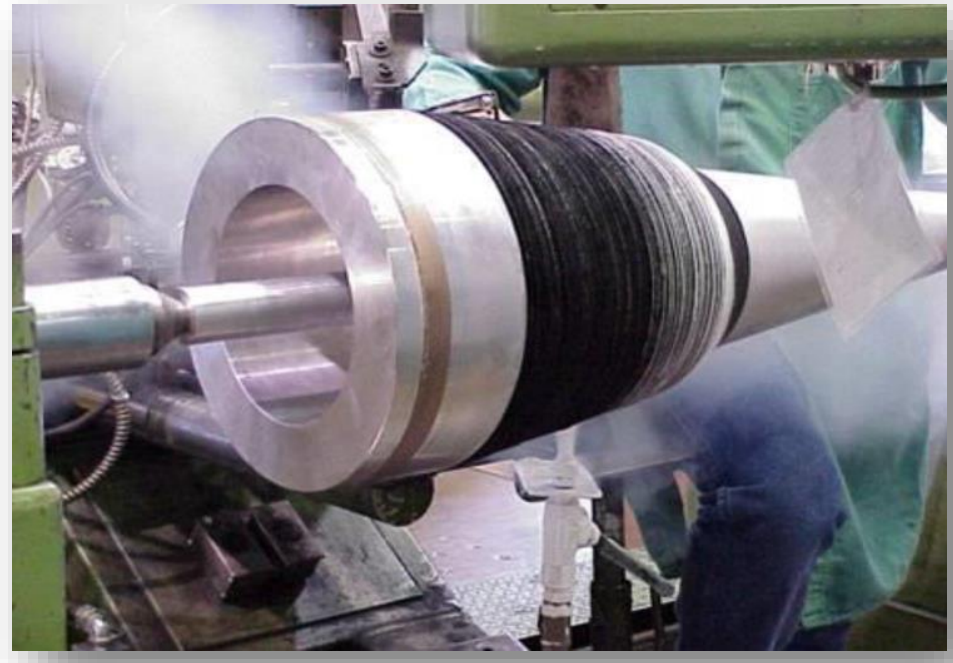
barrier systems in long duration, multiple start/stop tests. Oxidation protection systems provided by:

- COIC -- 3 systems

- Exothermics -- 1 system

- Plasma Processes -- 2 systems

- Seven nozzle extensions manufactured and successfully tested in December 2014.

- Additional testing in Aug-Sep 2016.

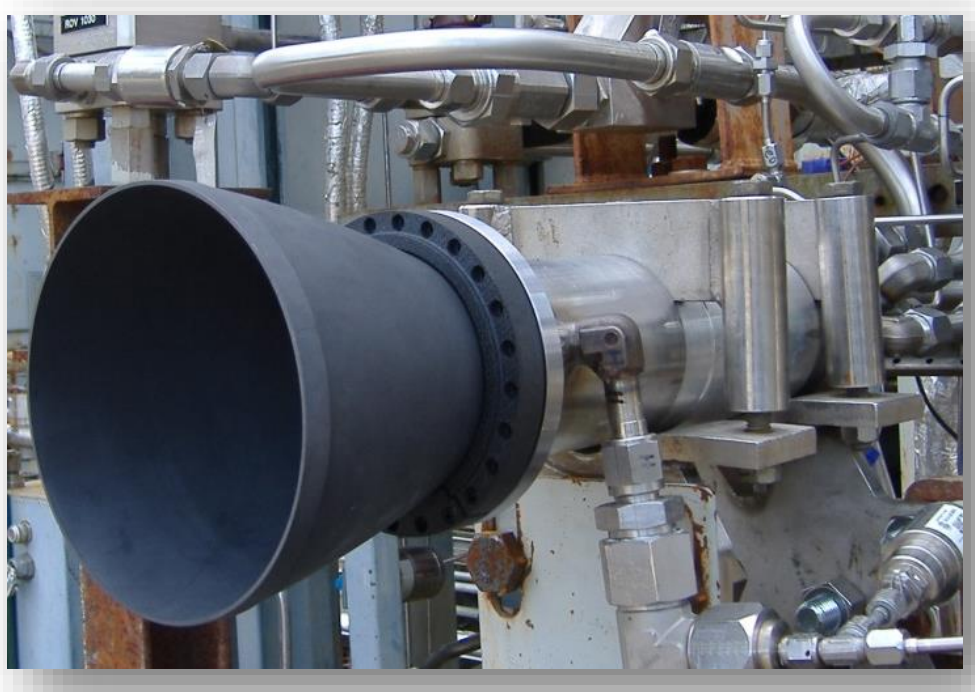




\section{Joint MSFC / Carbon-Carbon Advanced Technologies (C-CAT) effort}

- Demonstrate SiC coated C-C, which is being considered for LOX/LH2 nozzle extension applications

- Experimental enhanced-matrix C-C (EMCC) systems that do not require use of high-cost protective coatings

\section{Four 2D C-C Materials Tested}

A. ACC- 6 with silicon carbide (SiC) pack cementation coating

B. ACC- 6 with $\mathrm{SiC}$ enhanced matrix - an experimental material

C. ACC-4 with no coating

D. ACC- 6 with zirconium diboride (ZrB2) plus hafnium carbide (HfC) enhanced matrix - an experimental material

\section{CCNE's Prior to} Hot-Fire Testing

- All use T-300 PAN 3K heat treated material

- All used the same tooling.

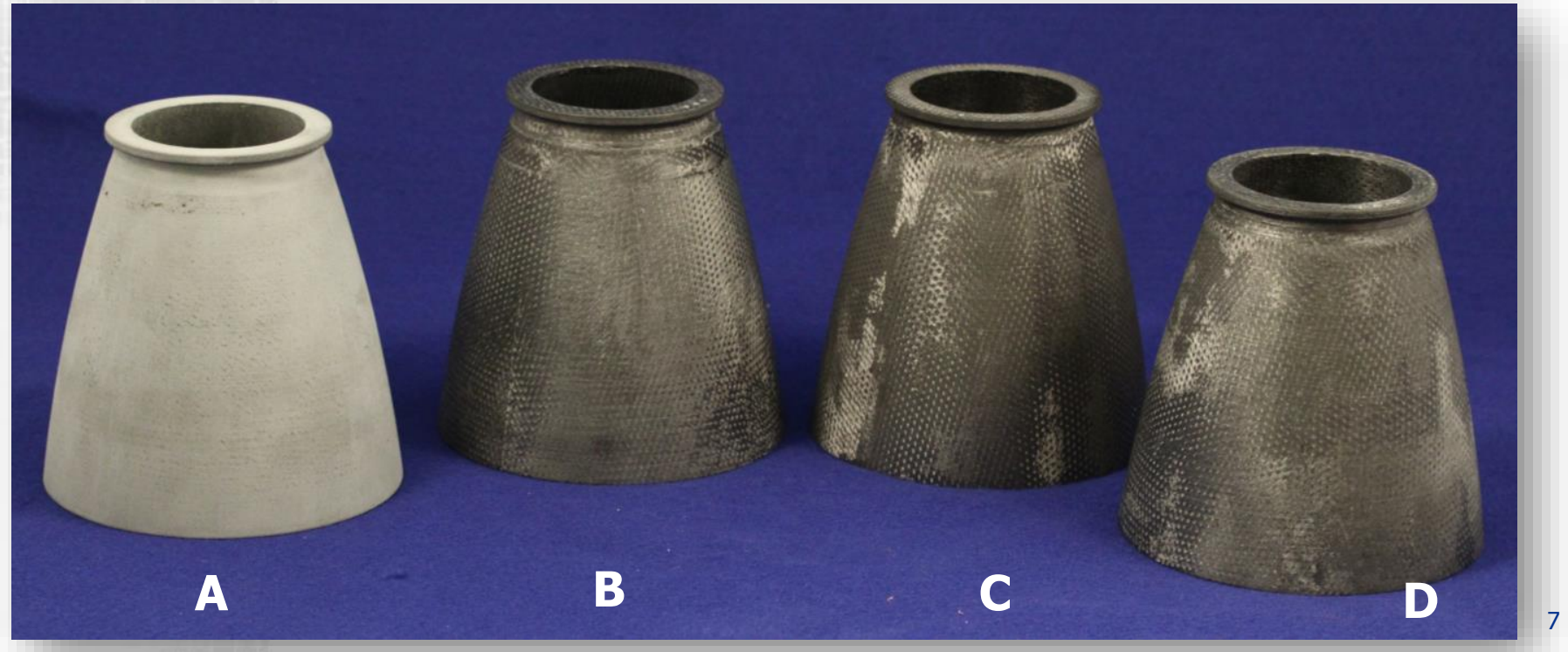




\section{NASA Extension Design and Chamber Interface (C-CAT)}

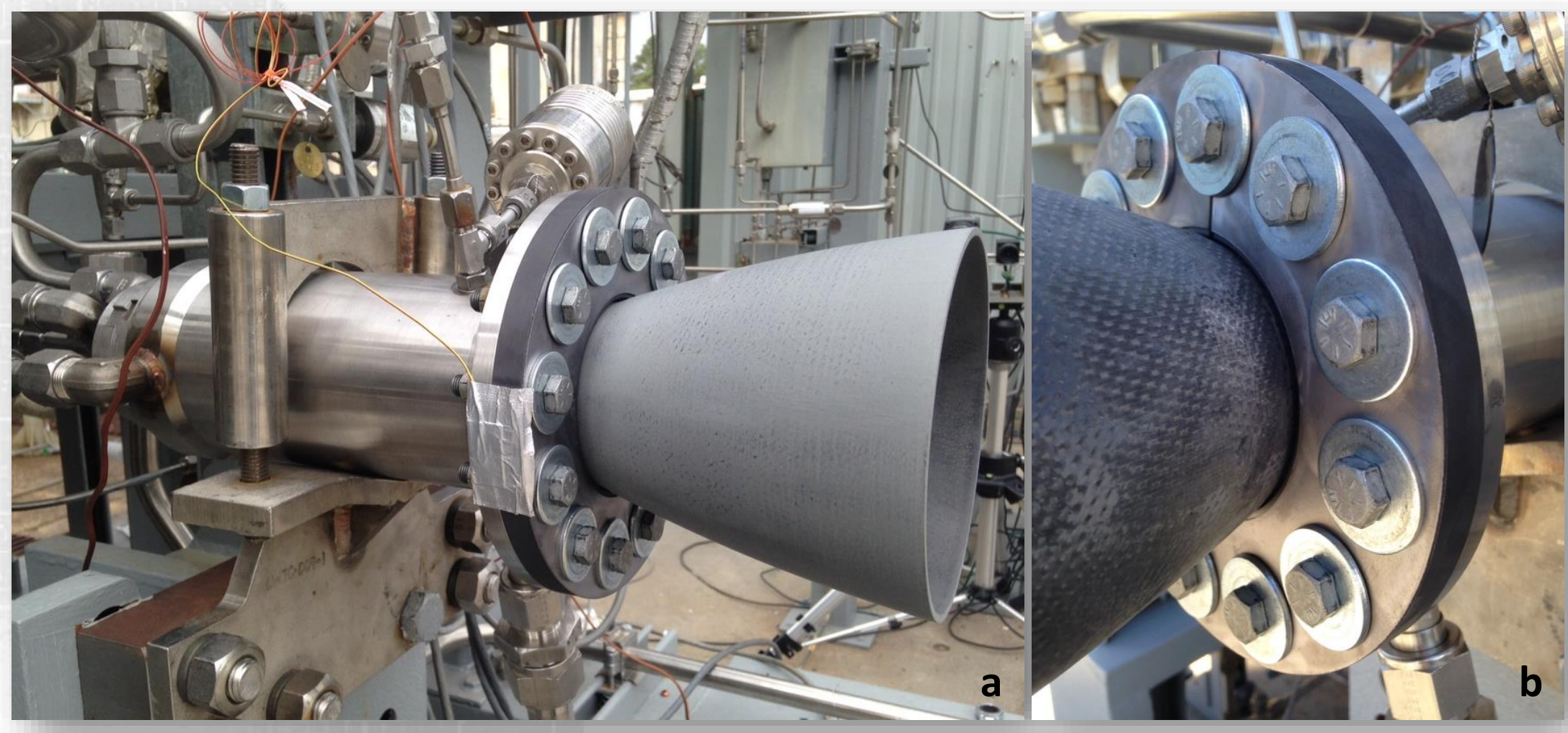

\section{Nozzle Extension Installed on Thrust Chamber Assembly}

a. Full assembly at MSFC TS- 115 .

b. View of tantalum backer split ring, graphite split ring, and overall interface region.

- C-C extensions attached to aft flange of combustion chamber using GES Graphite (PFI-25 and PFI-45) split rings.

- Grafoil, grade GTB flexible graphite, 0.060 " thick compressed seal at interface between graphite and combustion chamber flange.

- Tantalum split-ring backer plate at aft end of graphite split ring. 


\section{C-C Extension Hot-fire Testing Results}
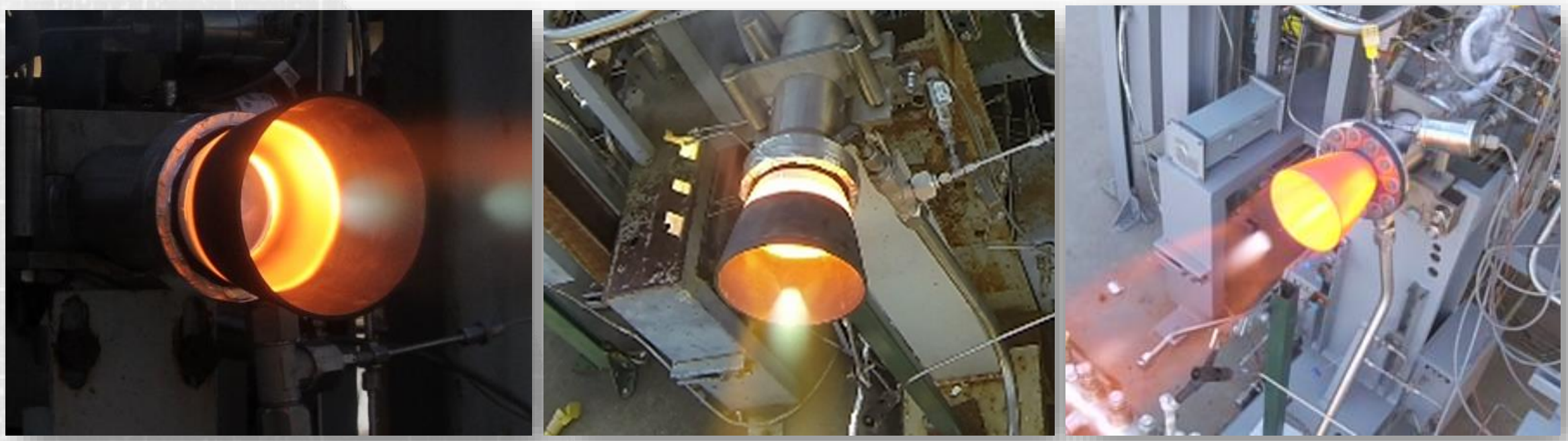

Orbital ATK Extension Test with COIC SiC + Hf

C-CAT Extension Test, ZrB2/HfC EMCC

\begin{tabular}{llc}
\hline \multicolumn{1}{c}{ Base Material } & \multicolumn{1}{c}{$\begin{array}{c}\text { Anti-Oxidation Protection } \\
\text { coating }\end{array}$} & $\begin{array}{c}\text { Accumulated Duration } \\
\text { sec }\end{array}$ \\
\hline OATK TW Rapid Densification 3 Cycles & Bare & 10 \\
OATK TW Rapid Densification 3 Cycles & COIC-SiC, No Filler & 90 \\
OATK TW Rapid Densification 3 Cycles & PPI ZrB2+SiC, APS & 30 \\
OATK TW Rapid Densification 3 Cycles & Exothermics Si-Partial SiC & 155 \\
OATK TW Rapid Densification 3 Cycles & PPI MoSi2-based, VPS & 30 \\
OATK TW Rapid Densification 3 Cycles & COIC-SiC + Hf-based Filler & 720 \\
OATK TW Rapid Densification 3 Cycles & COIC-SiC + Zr-based Filler & 480 \\
C-CAT 40 ACC-4 & Bare & 240 \\
C-CAT 40 ACC-6 & SiC Conversion & 2050 \\
C-CAT EMCC ACC-6 & None, SiC-enhanced resin EMCC & 10 \\
C-CAT EMCC ACC-6 & ZrB2/HfC enhanced matrix EMCC & 64 \\
\hline \hline
\end{tabular}



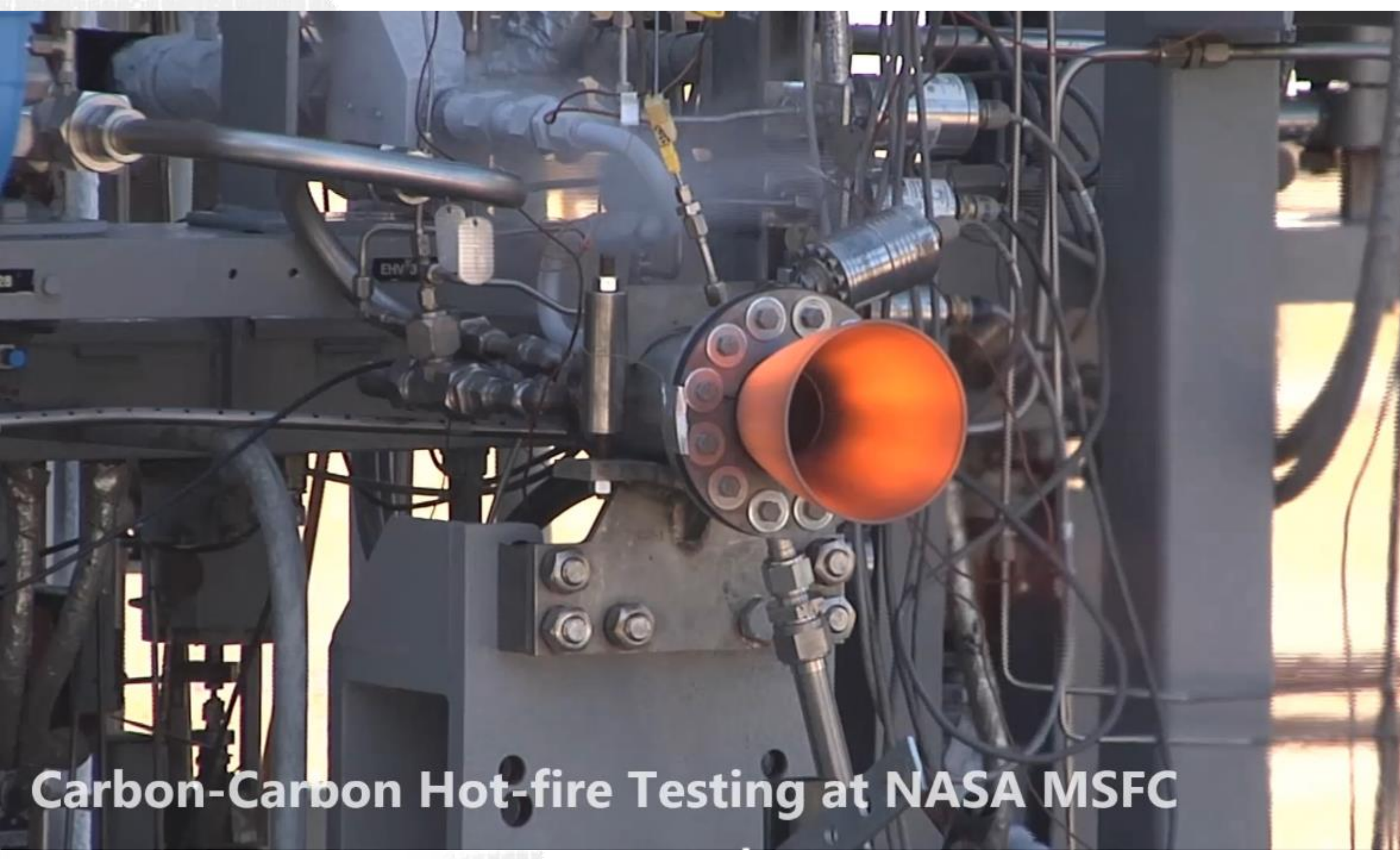

Carbon-Carbon Hot-fire Testing at NASA MSFC 


\section{NASA Orbital ATK Extension, COIC Hf-based filler}

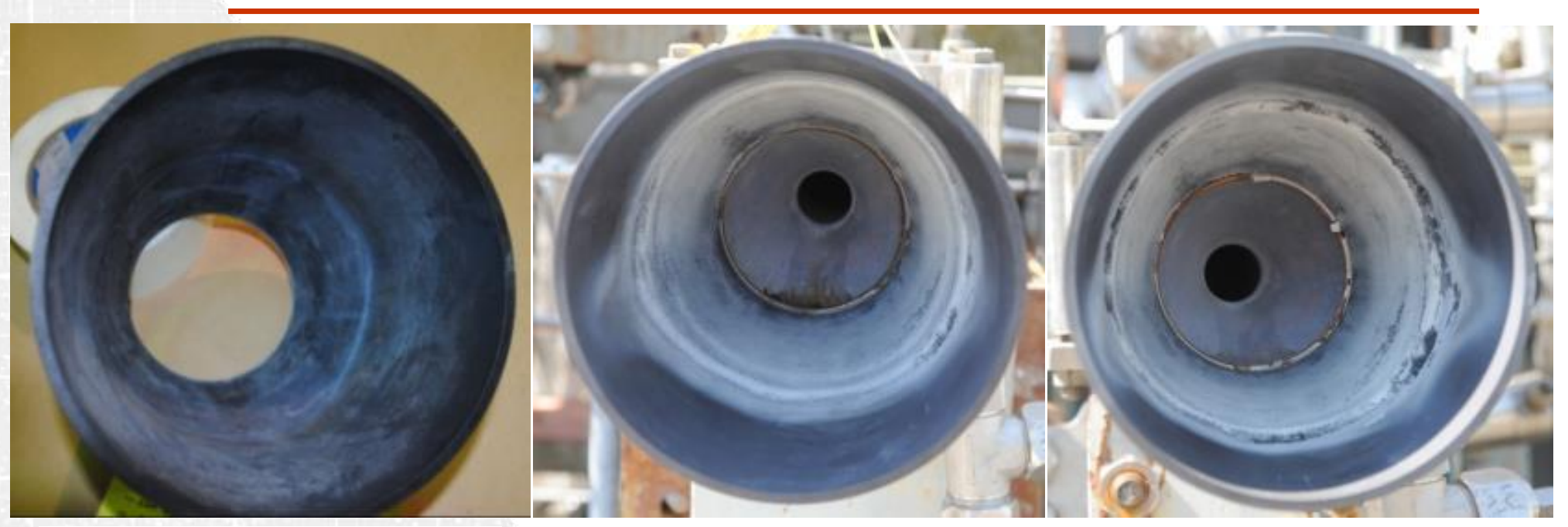

3 Starts, Post-120 sec

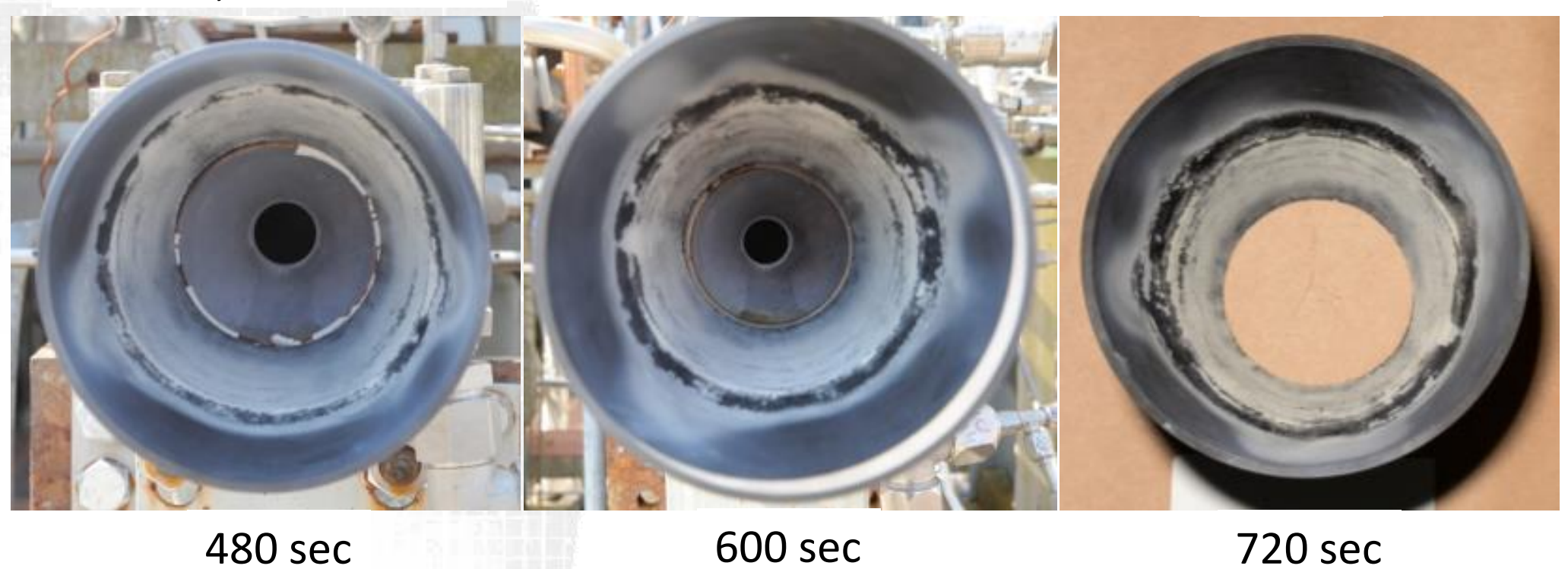

$<23 \%$ weight loss at elevated mixture ratios, although attributed to flow separation region as predicted 


\section{C-CAT ACC-6 with silicon carbide (SiC) coating}
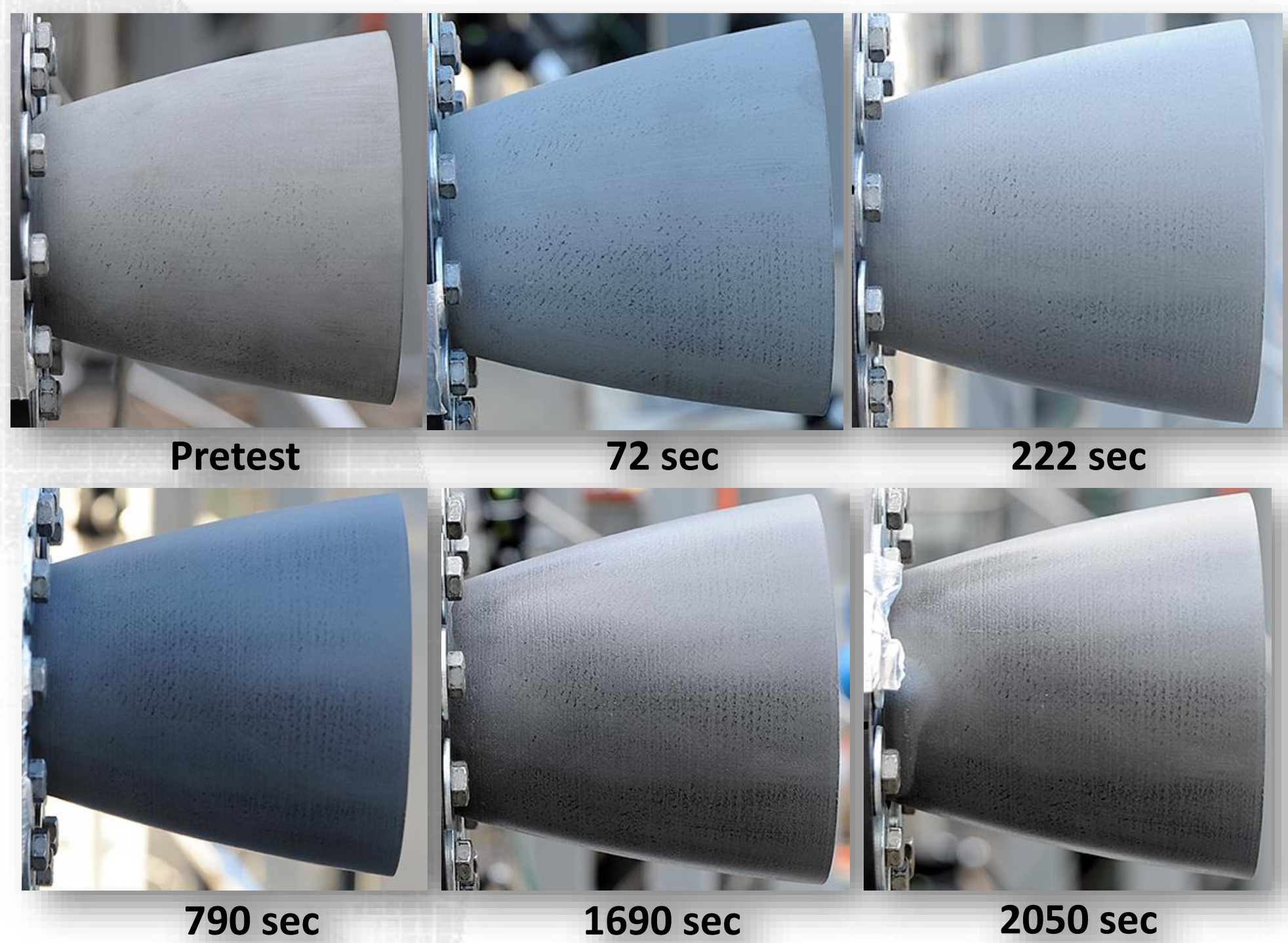

No erosion observed on ID surface; Note: oxidation more prevalent on OD aft end due to entrainment flow; based on results from EMCC material and uncoated testing 


\section{NASA Infrared Thermography during C-CAT Testing}

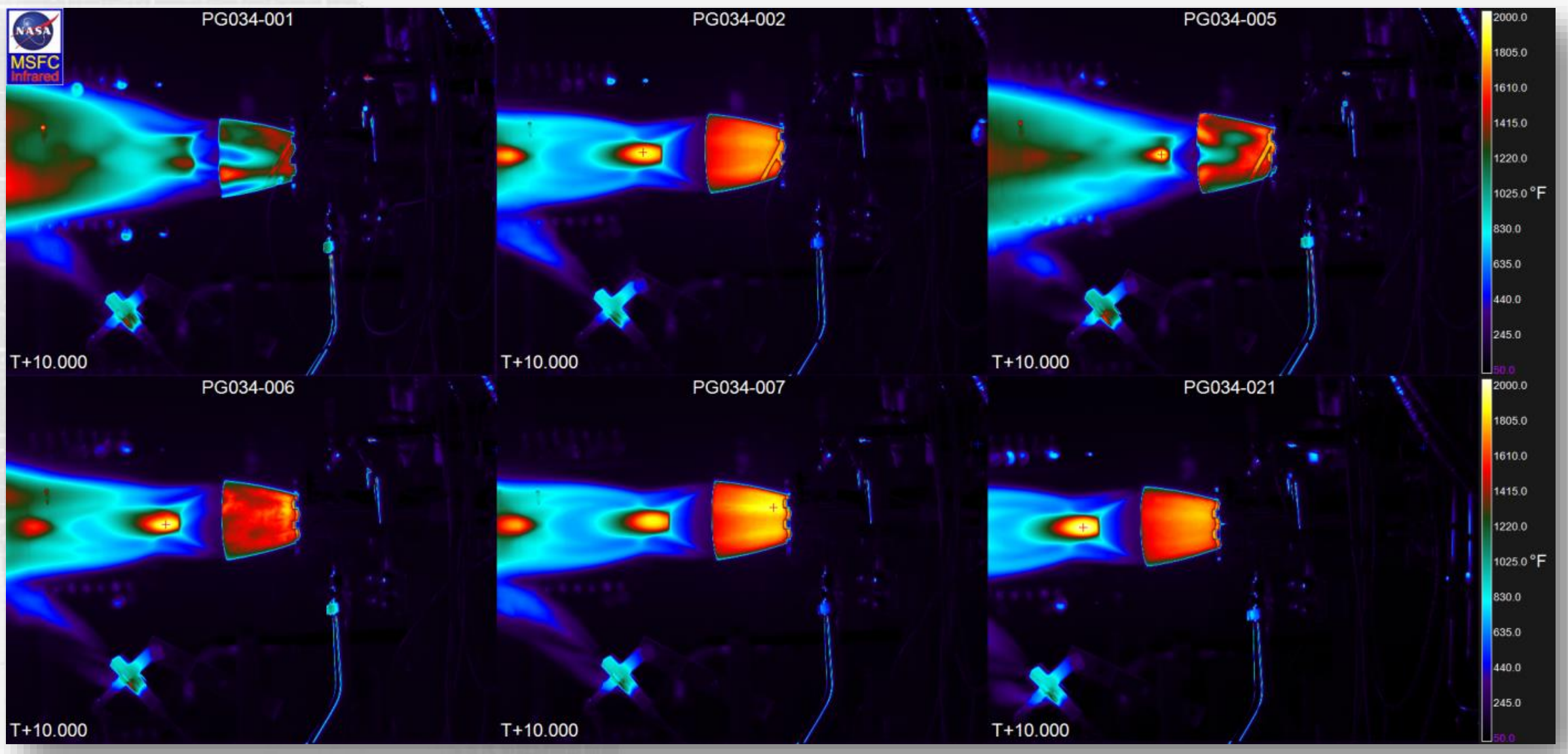

Comparison of infrared (IR) thermography imaging for C-CAT extensions at start +10 seconds with various amounts of streaking observed.

- Ply lifts observed in EMCC

- Operated at temperatures up to $2400^{\circ} \mathrm{F}$

Note: Tests $-002,-007,-021$ are with the SiC conversion coating. 


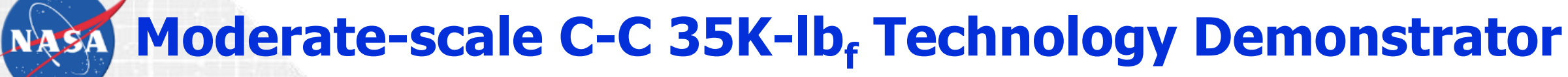

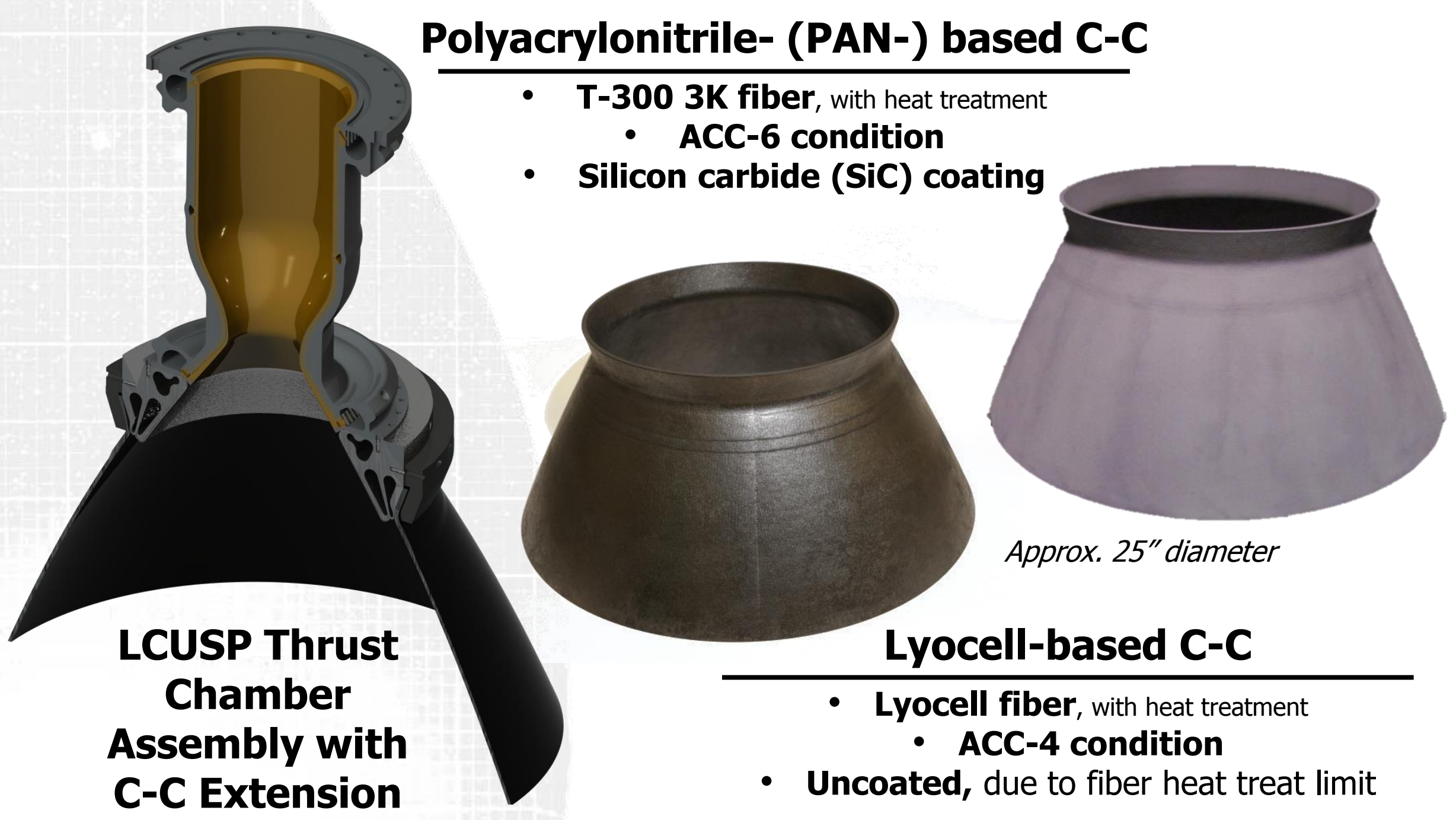

- Both extensions fabricated using the same tooling 


\section{C-C Subelement and Coupon Testing}

- Tag-end rings sectioned from 35K demonstrator extensions

- Developed NDE techniques for C-C extensions

- Coupon material testing (axial compression, interlaminar tension, hoop thermal expansion)

- Hydrostatic loading of conical ring full diameter sections
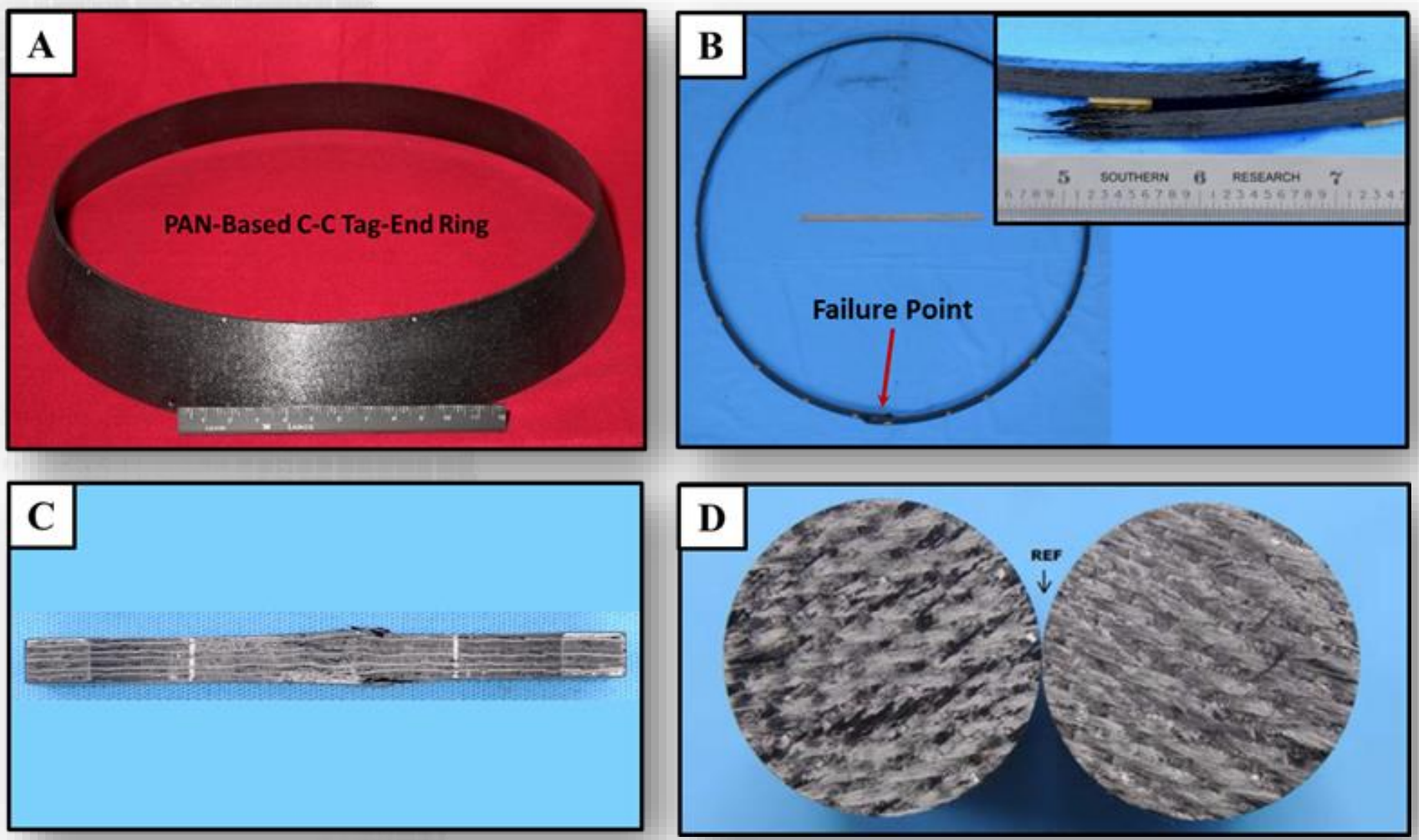
- Optical non-contact measurement development supporting $\mathrm{C}-\mathrm{C}$ development

- Using digital image correlation (DIC) to obtain full field surface strains and displacements

- Elevated temperatures during hot-fire testing using visible wavelength (DIC) caused issues during transients

- Evaluating alternate DIC techniques such as UV-DIC

- DIC techniques have been proven during full-scale lab testing
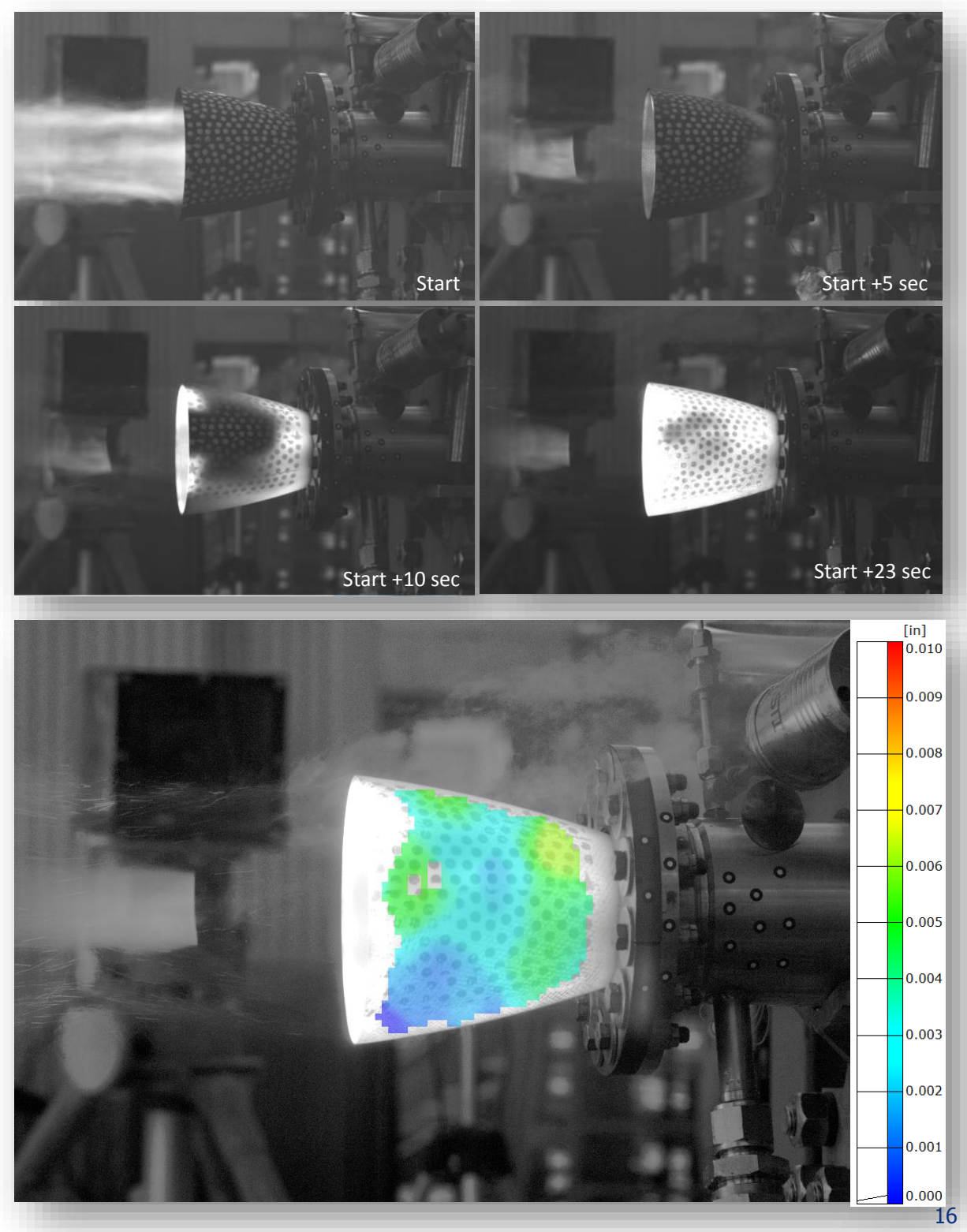
- NASA and its commercial space partners are interested in advancing a domestic commercial supply chain for Carbon-Carbon Nozzle Extensions (CCNE's).

- MSFC is interested in evaluating materials appropriate for cryogenic upper stage engines and obtaining preliminary hot-fire test data in relevant environments.

- C-C nozzle extension efforts have proceeded primarily through the following:

- Small business contracts investigating: attachment concepts, material systems, etc.

- MSFC in-house technology development projects:

- C-C material systems, databases, advancement of technology and material readiness levels (TRL, $M R L)$, geometry effects on properties for flat vs. complex shapes, etc.

- Materials screening with $1.2 \mathrm{~K}-\mathrm{Ib}_{\mathrm{f}} \mathrm{LOX} / \mathrm{GH} 2$ thruster to obtain preliminary hot-fire test data.

- Completed testing on variety of materials from C-CAT and Orbital ATK.

- Moderate-size demos via $35 \mathrm{~K}-\mathrm{Ib}_{\mathrm{f}}$ LOX/LH2 engine - low-budget feasibility assessments.

- Extended duration subscale testing has demonstrated extension and coating technology

- C-CAT PAN ACC-6 w/ SiC Conversion Coating = 2,050 sec hot-fire

- Orbital ATK Tape Wrap w / COIC Hf-based filler $=720 \mathrm{sec}$ hot-fire

- NASA MSFC to complete testing of $35 \mathrm{k}-\mathrm{lb}_{\mathrm{f}}$ truncated extensions on 3D printed copper chamber in Fall-2017 


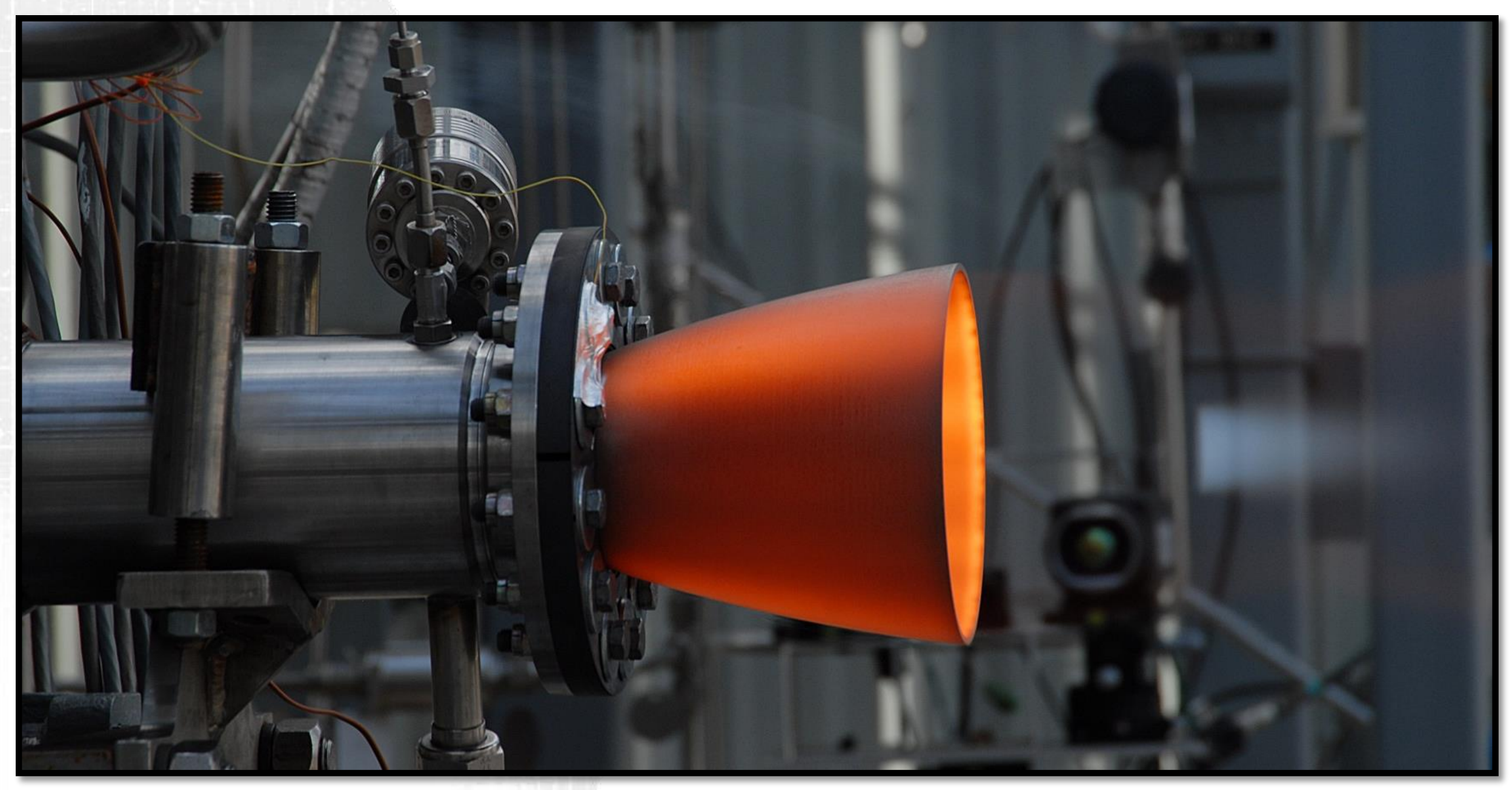




\section{Acknowledgements}

- Sandy Elam Greene

- Cynthia Sprader

- David Olive

- Test Stand 115 Crew

- Will Brandsmeier

- Cory Medina

- Jennifer Adams

- Ian Johnston

- Gary Kelly

- Van Luong

- Gregg Jones

- Chris Protz

- Derek Moody
- Darrell Gaddy

- Brian Sullivan / MR\&D

- Leslie Weller / MR\&D

- David Myers /EM20

- James Walker / EM20

- Ken Cooper

- John Fikes

- Tony Kim

- Steve Fentress / Rocketdyne

- Matt Crisanti / C-CAT

- James Thompson / C-CAT

- Aaron Brown / C-CAT

- John Shigley / OATK
- $\quad$ Robert Roberts / OATK

- Hank Dovey / OATK

- John Koenig

- Jacques Cuneo

- Chanse Appling

- Steve Fentress

- Bill Marshall / GRC

- Southern Research

- C-CAT

- Orbital ATK

- Materials Research and Design (MR\&D) 


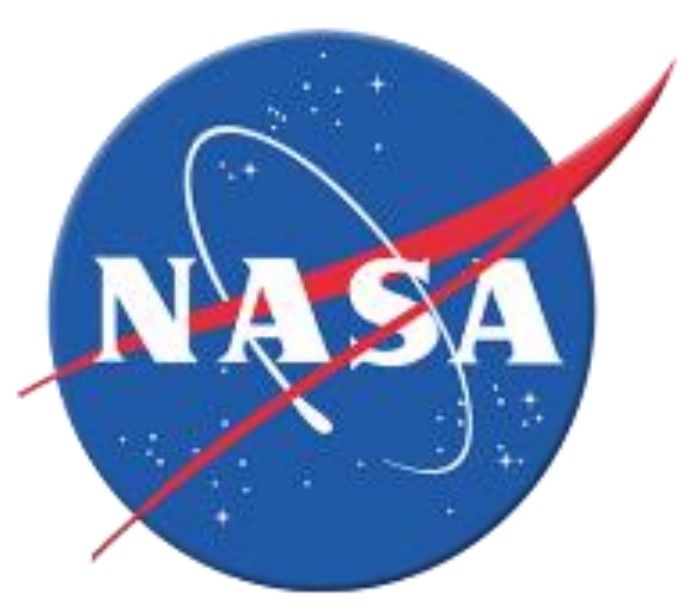

Contact: Paul Gradl NASA MSFC 256.544.2455

Paul.R.Gradl@nasa.gov 